\title{
Axin, the classic scaffold protein of Wnt/ $\beta$-catenin signalling as a potential drug-target for vitiligo
}

Duozhi Chen ${ }^{1}$, Shirui Fan², Xueying Lu³, Chenxu Jing ${ }^{2}$, Deng Zang ${ }^{3}$, Bijuan Yang ${ }^{1}$, Jieyun $\mathrm{Cai}^{1}$, Yiting Wang ${ }^{2}$, Xinfang Zhang ${ }^{2}$, Lin $\mathrm{Li}^{4}$, Haji Akber Aisa ${ }^{3}$, and Xiaojiang Hao ${ }^{2}$

${ }^{1}$ Kunming Institute of Botany Chinese Academy of Sciences

${ }^{2}$ State Key Laboratory of Phytochemistry and Plant Resources in West China, Kunming Institute of Botany, Chinese Academy of Sciences

${ }^{3}$ Xinjiang Technical Institute of Physics and Chemistry, Chinese Academy of Sciences

${ }^{4}$ Shanghai Institute of Nutrition and Health, Chinese Academy of Sciences

November 19, 2020

\begin{abstract}
Background and Purpose: Humans have been fighting vitiligo for centuries but still being inferior due to the lack of efficiency drugs and therapies. While some research has implied the therapeutic potential of $\mathrm{Wnt} / \beta$-catenin signalling on curing vitiligo but correlation mechanism is not clear and no Wnt-specific anti-vitiligo drug has been reported. Here, We identified how vitiligo could be treated by regulating Wnt and two lead compounds of new anti-vitiligo drugs have been found. Experimental Approach: Wnt agonists were rational synthesized and then be evaluated their effects on vitiligo in B16 cells and C57B/L mouse. Furthermore, Co-IP and Site-directed mutagenesis were employed to indicate the mechanism and the target of the compounds. Key Results: HCJA121 and HCJA404 could significantly promote the synthesis of melanin, restore the pigmented function of skin, and improve the symptoms of vitiligo. Mechanism studies indicated that HCJA121 and HCJA404 target the DAX domain of Axin by binding to LYS781 and LEU784 then potentiate the Axin-LRP6 association and eventually promoted melanogenesis. Conclusions and Implications: These findings imply an alternative regulatory mechanism of melanogenesis and the Axin protein could be a new target for anti-vitiligo agents which reveal a therapeutic strategy for vitiligo. Besides, HCJA121 and HCJA404 may represent potential compounds for vitiligo treatment.
\end{abstract}

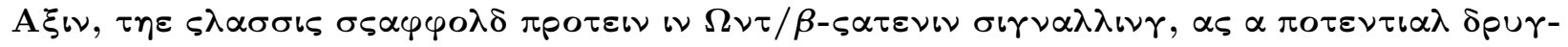

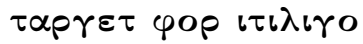

Duo-zhi Chen ${ }^{1,+, *}$, Shi-rui Fan ${ }^{1,2},+$, Xue-yin Lu ${ }^{3,+}$, Chenxu Jing ${ }^{1}$, Den Zang ${ }^{3}$, Bijuan Yang ${ }^{1,2}$, Jie-yun $\mathrm{Cai}^{1}$, Yi-ting Wang ${ }^{1,2}$, Lin $\mathrm{Li}^{4 *}$, Haji Akber Aisa ${ }^{3, *}$, and Xiaojiang Hao ${ }^{1, *}$

${ }^{1}$ State Key Laboratory of Phytochemistry and Plant Resources in West China, Kunming Institute of Botany, Chinese Academy of Sciences, Kunming 650201, China

${ }^{2}$ University of Chinese Academy of Sciences, Beijing 100049, China.

3 The Key Laboratory of Plant Resources and Chemistry of Arid Zone, Xinjiang Technical Institute of Physics and Chemistry, Chinese Academy of Sciences, Urumqi 830011, China

${ }^{4}$ Shanghai Institute of Nutrition and Health, Chinese Academy of Sciences, Shanghai, 200031, China

+ These authors contributed equally to this work.

* Corresponding author 
E-mail address : haoxj@mail.kib.ac.cn. (X. H. Hao); haji@ms.xjb.ac.cn (A. A. Haji); lli@sibs.ac.cn. (L. Li); chenduozhi@mail.kib.ac.cn (D. Z. Chen),

\begin{abstract}
Background and Purpose: Humans have been fighting vitiligo for centuries; however, there remains a lack of efficient therapies. While research has implied the therapeutic potential of Wnt/ $\beta$-catenin signalling for curing vitiligo, the mechanism underlying this correlation is unclear, and no Wnt-specific anti-vitiligo drug has been reported. Here, we determined a treatment approach for vitiligo that acts through regulation of Wnt and identified two new lead compounds for anti-vitiligo treatment.
\end{abstract}

Experimental Approach: Wnt agonists were rationally synthesized and evaluated for their effects on vitiligo in B16 cells and C57B/L mice. Furthermore, co-immunoprecipitation and site-directed mutagenesis were employed to determine the mechanism and target of the compounds.

Key Results: HCJA121 and HCJA404 could significantly promote the synthesis of melanin, restore the pigmented function of skin, and improve the symptoms of vitiligo. Mechanistic studies indicated that HCJA121 and HCJA404 target the DAX domain of axin via binding to LYS781 and LEU784, potentiating the axinLRP6 association and eventually promoting melanogenesis.

Conclusions and Implications: These findings imply an alternative regulatory mechanism of melanogenesis, and the axin protein could be a new target for anti-vitiligo agents, revealing a therapeutic strategy for vitiligo. Besides, HCJA121 and HCJA404 may represent potential candidates for vitiligo treatment.

\title{
Keywords
}

vitiligo, phenanthridine alkaloids, melanogenesis, Wnt/ $\beta$-catenin signalling, axin,

\section{Abbreviations}

8-MOP: 8-methoxypsoralan

DMSO: dimethylsulfoxide

L-DOPA: L-3,4-dihydroxyphenylalanine

MITF: melanocyte-inducing transcription factor

TYRP: tyrosinase-related protein

TYR: tyrosinase

co-IP: co-immunoprecipitation

MDA: malondialdehyde

\section{Bullet point summary}

\section{What is already known}

Wnt $/ \beta$-catenin signalling pathway plays an important role in melanocyte expansion and melanogenesis.

Regulation of Wnt/ $\beta$-catenin signalling has therapeutic potential for vitiligo treatment.

\section{What this study adds}

Identification of a new Wnt-associated regulatory mechanism of melanogenesis and axin protein as a new target for anti-vitiligo treatment.

This study identified two potent anti-vitiligo agents.

\section{Clinical significance}

This work reveals a new therapeutic strategy for vitiligo. 
HCJA121 and HCJA404 may represent potential candidates for vitiligo treatment.

\section{Introduction}

Vitiligo is an acquired chronic depigment disorder of the skin resulting from the selective destruction of melanocytes (Boniface, Seneschal, Picardo \& Taieb, 2018; Ezzedine, Eleftheriadou, Whitton \& van, 2015; Picardo et al., 2015). It is a high morbidity disease that equally affects adults and children of both sexes. Vitiligo is prevalent in $0.51 \%$ of the world's population; however, in some countries, this number is significantly higher: 8.8\% (India) and 4\% (Mexico) (Boisseau-Garsaud, Garsaud, Cales-Quist, Helenon, Queneherve \& Claire, 2000; Ezzedine et al., 2012; Sehgal \& Srivastava, 2007). Vitiligo is primarily classified as nonsegmental vitiligo and segmental vitiligo. Non-segmental vitiligo, which is characterized by symmetrical and bilateral white patches, is the most common form of this disease; segmental vitiligo is considerably less common and usually has a unilateral distribution.

Although vitiligo is not a fatal disease, it is associated with considerable negative social effects among patients, especially women and children (Elbuluk \& Ezzedine, 2017; Olsen, Gallacher, Finlay, Piguet, \& Francis, 2016; Anonymous, 2015). Therefore, research on effective treatment of vitiligo has never stopped; interventions developed for vitiligo include topical therapies, phototherapy therapies, herbal medicine, surgical treatments, and psychological treatments. Medications such as glucocorticosteroids, calcineurin inhibitors, melagenina, vitamin D, and Janus kinase inhibitors have been employed for improving the symptoms of vitiligo. Unfortunately, the outcomes of the aforementioned treatments vary among individuals, and they are often unsatisfactory; furthermore, treatment is more efficient in recently developed lesions than in older lesions (Hayashi et al., 2016; Lopes, Trevisani, Trevisani, Trevisani, Melnik \& Melnik, 2016; Cohen, Elbuluk, $\mathrm{Mu} \&$ Orlow, 2015; Niezgoda et al., 2017). Therefore, there is a need for the development of treatment strategies for vitiligo, including the identification of new anti-vitiligo chemotherapeutic agents (Rashighi \& Harris, 2017; Rodrigues, Ezzedine, Hamzavi, Pandya \& Harris, 2017).

Previous studies have discussed the role of the Wnt $/ \beta$-catenin signalling pathway in the development of melanocytes (Li et al., 2019; Yamada et al., 2013). Wnt/ $\beta$-catenin, a highly conserved signalling pathway, is important for development, particularly, embryonic development. Aberrant Wnt signalling is involved in many diseases (Clevers \& Nusse, 2012; Zeng et al., 2005; Zimmerman, Moon \& Chien, 2012). Previous studies have suggested that regulation of Wnt could be used to treat vitiligo through modulation of melanocyte functions, and appropriate Wnt agonists may play an effective role in curing vitiligo. However, perhaps because of the lack of effective and Wnt-specific agonists for medical applications, the effect of Wnt-specific small-molecule agonists on vitiligo have not been proven thus far.

Our previous research identified a new series of phenanthridine-specific agonists of the Wnt/ $\beta$-catenin signalling pathway (Wang et al., 2013; Chen et al., 2018; Chen et al., 2016; Chen et al., 2019). In consideration of the role of Wnt in melanogenesis, it is worth discussing whether appropriate, potent phenanthridine Wnt agonists have the potential to cure or improve the symptoms of vitiligo. Therefore, in this study, we designed new phenanthridine derivatives and evaluated their anti-vitiligo effects in vitro and in vivo. Furthermore, we revealed an efficient regulatory network underlying melanin biosynthesis and a related target protein. These results suggest a promising therapeutic strategy for vitiligo and the potential applications of phenanthridine derivatives in the treatment of vitiligo.

\section{Materials and Methods}

\section{Cell culture}

The murine B16 melanoma cell line was purchased from the Type Culture Collection of the Chinese Academy of Sciences (Shanghai, China). The B16 cells were cultured in HG-DMEM (Gibco Life Technologies, Waltham, MA, USA). Wnt3a was added $(2 \mathrm{ng} / \mathrm{mL})$ to the media, followed by supplementation with $10 \%$ FBS (BI, Biological Industries), penicillin G $(100 \mathrm{U} / \mathrm{mL})$, and streptomycin (100 $\mu \mathrm{g} / \mathrm{mL}$ ) (Gibco-BRL, Grand Island, NY, USA). The cells were maintained in a humidified incubator under $5 \% \mathrm{CO}_{2}$ at $37{ }^{\circ} \mathrm{C}$, and they were sub-cultured every 2 days to maintain logarithmic growth. 


\section{Tyrosinase activity}

Cellular tyrosinase activity was estimated by measuring the rate of L-3,4-dihydroxyphenylalanine (L-DOPA) oxidase activity (Kim et al., 2013). B16 cells were seeded in a 6 -well plate at a density of $2.5 \times 10^{5}$ cells/well. After $24 \mathrm{~h}$ of incubation, the cells were treated with different concentrations of the test compounds for 24 $\mathrm{h}, 8$-MOP was added at $50 \mu \mathrm{M}$ as a positive control, and $2 \mu \mathrm{L}$ of DMSO was added to the control group. Cells were then lysed with PBS containing 1\% Triton X-100 and 1\% sodium deoxycholate for 30 min at -20 ${ }^{\circ} \mathrm{C}$. The lysates were centrifuged at $12,000 \times \mathrm{g}$ for $15 \mathrm{~min}$. A reaction mixture containing $90 \mu \mathrm{L}$ of each cell lysate and $10 \mu \mathrm{L}$ of $10 \mathrm{mM}$ L-DOPA was added to each well in a 96-well plate. After incubation for 20-60 min (according to the amount of dopachrome formed) at $37^{\circ} \mathrm{C}$ in the dark, the product dopachrome was detected at $490 \mathrm{~nm}$ using a multi-plate reader (Spectra Max M5/M5e). The tyrosinase activity of each sample was calculated relative to that of the control group and corrected for the protein concentrations, considering the control group as representing $100 \%$ activity.

\section{Melanin content measurement}

B16 cells were seeded in a 6 -well plate at a density of $1.8 \times 10^{5}$ cells/well. After $24 \mathrm{~h}$ of incubation, the cells were treated with different concentrations of the test compounds for $48 \mathrm{~h}, 8$-MOP was added at 50 $\mu \mathrm{M}$ as a positive control, and $2 \mu \mathrm{L}$ of DMSO was added to the control group. Subsequently, B16 cells were washed twice with PBS (pH 7.4) and lysed in $100 \mu \mathrm{L}$ of RIPA lysis buffer (AR0105-100) (BOSTER Biological Technology, Wuhan, China) for $40 \mathrm{~min}$ at $4{ }^{\circ} \mathrm{C}$. The lysates were centrifuged at $12,000 \times \mathrm{g}$ for $20 \mathrm{~min}$ at $4{ }^{\circ} \mathrm{C}$. We collected $3 \mu \mathrm{L}$ of supernatant from the cell extracts to measure the total protein content using a BCA kit assay (PP02) (Biomed, Beijing, China). The pellets were treated with $190 \mu \mathrm{L}$ of $1 \mathrm{M} \mathrm{NaOH}$ (with 10\% DMSO) for $1 \mathrm{~h}$ at $80{ }^{\circ} \mathrm{C}$, and the optical density was detected at $405 \mathrm{~nm}$ using a multi-plate reader (SpectraMax M5/M5e). The melanin content was calculated relative to that in the control group and corrected for the protein concentrations, considering the control group as representing $100 \%$ melanin content.

\section{Western blot analysis}

The protein samples were prepared from B16 cells as described above and separated via 10\% SDS-PAGE. The gels were transferred to PVDF membranes, blocked with $5 \%$ skim milk or $5 \%$ BSA, and incubated overnight at $4{ }^{\circ} \mathrm{C}$ with appropriate antibodies. Following incubation with the secondary antibody, the protein bands were detected using an enhanced chemiluminescence (ECL) western blotting detection kit, and the signals were quantified using a ChemiDoc MP Imaging system (Bio-Rad Laboratories Inc. Hercules, CA, USA). All experiments were performed five times.

\section{Establishment of vitiligo model and treatment regimen}

C57BL/6 mice (age 4-7 weeks; body weight 18-38 g;) with black dorsal skin were supplied by Vital River Laboratory Animal Technology Co. Ltd., Beijing (Approval ID: SCXK-(jing) 2014-0013). Four to six mice were housed in clear plastic cages $(225 \times 340 \times 155 \mathrm{~mm})$ containing bedding made of paper/cellulose with ad libitum access to standard chow diet and water. All animals were housed at $22 \pm 4{ }^{\circ} \mathrm{C}$ and $50 \pm 10 \%$ humidity. The experiments were approved by the Institutional Ethical Committee for Laboratory of Xinjiang Medical University (Approval ID: SYXK-(xin) 2016-0002) and conducted in accordance with relevant guidelines and regulations. The dorsal side of the animals was shaved a day before the experiment using electric clippers. Dorsal hypopigmentation in mice was induced by hydroquinone (HQ) as previously described. Briefly, a 2 $\mathrm{cm} \times 2 \mathrm{~cm}$ depilated region of each mouse was smeared with $0.5 \mathrm{~mL}$ of $5 \%$ HQ (CAS:23-31-9) once a day for 50 days. The animals were acclimatized for 1 week and randomly divided into six groups $(\mathrm{n}=12$ per group): (1) the control group, in which distilled water was administered and smeared; (2) the vitiligo model group, which received $5 \% \mathrm{HQ}$ (Jiang lai) on the $2 \times 2 \mathrm{~cm}$ shaved dorsum for 50 days and distilled water for 30 days; (3) the 8-MOP group, which received 5\% HQ for 50 days and 8-MOP (4.25 mg/kg) for 30 days; (4) -(6) the HCJA121 group, which received 5\% HQ for 50 days and HCJA121 at various concentrations $(0.0425 \mathrm{mg} / \mathrm{kg}(\mathrm{n}=12), 0.425 \mathrm{mg} / \mathrm{kg}(\mathrm{n}=12), 4.25 \mathrm{mg} / \mathrm{kg}(\mathrm{n}=12))$ for 30 days; and $(7)-(9)$ the HCJA404 group, which received $5 \%$ HQ for 50 days and HCJA121 at the same doses specified above. At the end of the 
30-day treatment, the blood samples were collected retro-orbitally from the mice. In addition, dorsal skin samples were isolated from the animals.

\section{Macroscopic assessment}

The extent of depigmentation was evaluated in terms of the percentage of affected area, as described previously, and was scored as follows: 0 , no depigmentation (0\%); $1.0-10 \%$ depigmented area; $2,10 \%-25 \% ; 3$, $25 \%-75 \% ; 4,75 \%-100 \%$; and $5,100 \%$. The final vitiligo score of each experimental group was calculated as the average for individual mice.

\section{Enzyme-linked immunosorbent assay (ELISA)}

We measured the levels of interleukin 6 (IL-6), tumour necrosis factor- $\alpha$ (TNF- $\alpha$ ), tyrosinase (TYR), monoamine oxidase (MAO), and malondialdehyde (MDA) in the mouse or guinea pig sera using specific ELISA kits (NJJCBIO Co., Nanjing, China; Elabscience Biotechnology, China) according to the manufacturers' instructions. The absorbance at $450 \mathrm{~nm}$ was measured using a Spectra Max M5 microplate reader (Molecular Devices, San Diego, CA, USA).

\section{Histological examination}

H\&E staining was performed on skin sections as per standard protocols. The epidermis was measured as the distance from the basal layer to the surface of the epidermis. The melanin-containing hair follicles (HF) in the murine skin were evaluated by Lillie staining.

\section{Quantitative real-time PCR (qPCR)}

Total RNA of the PEDV-infected cells or recombinant plasmid-transfected cells was extracted using RNAiso Plus reagent (Takara, Japan) according to the manufacturer's protocols. The concentrations of the extracted RNA were measured using a Thermo Scientific Nano Drop 2000c system (Thermo Scientific, USA). Genespecific primers for qPCR are listed in Table S3. qPCR was performed using ChamQ Universal SYBR qPCR Master Mix on an ABI 7300 real-time PCR system. $\beta$-actin served as the endogenous control. The relative values for mRNA accumulation were determined using the $2^{-\Delta \Delta " \tau}$ method and compared with mock-treated results $^{22}$. We tested all compounds independently five times to obtain five samples $(\mathrm{N}=5)$, and each sample was run in triplicate.

\section{Statistical Analysis}

The data and statistical analysis comply with the recommendations of the British Journal of Pharmacology on experimental design and analysis in pharmacology (Curtis et al., 2018). All data were obtained from five independent experiments (i.e. not treating technical replicates as independent values) and expressed as means \pm S.E.M. WB and PCR data were normalized to the values of the control group (100\% or 1, respectively) and expressed as mean \pm S.E.M. Statistical analysis was undertaken only for studies where each group size was at least $\mathrm{n}=5$, where $\mathrm{n}=$ number of independent values. Animals were separated into groups randomly, and the groups were randomized into drug-treated or control group before treatments. For blinding, experimentation and analysis involved different persons. The total number of mice used in the present study was 108, all data are expressed as means \pm S.E.M. group sizes across all experiments were $\mathrm{n}=$ 12. Student's t test was used to compare the means of two groups, while statistical significance for multiple groups was determined by ANOVA. a $\mathrm{P}<0.05$ was considered statistically significant. $\left({ }^{*} P<0.05,{ }^{* *} P<\right.$ $0.01,{ }^{* * *} P<0.001$, and $\left.{ }^{* * * *} P<0.0001\right)$. All statistical analyses were carried out with GraphPad Prism 7.0 from GraphPad Software Inc., CA, USA. For statistical analysis, Prism(Version 5.0d, GraphPad Software, San Diego, CA, USA) was used.

\section{Results}

\section{Identification of phenanthridine derivatives Wnt agonists and their effects on melanin levels}

Phenanthridines have been identified as specific Wnt/ $\beta$-catenin signalling agonists. To identify more potent Wnt agonists, we designed and synthesized phenanthridine derivatives as Wnt agonists and performed studies 
on their structural optimization and structure-activity relationships (SAR). Thus, we identified additional potent Wnt agonists, including HCJA121, HCJA404, and compounds 1-6 (Fig. 1a, Fig. S1).

The effects of these eight phenanthridine derivatives on the levels of melanin were initially evaluated in B16-F10 cells treated with Wnt3a-conditioned media, using 8-methoxypsoralan (8-MOP) (Lerner, Denton \& Fitzpatrick, 1953) as a positive control. The absorbance of the same number of cells treated with phenanthridine derivatives at $50 \mu \mathrm{M}$ indicated that HCJA121 and HCJA404 strongly promoted melanin synthesis compared to 8-MOP (Fig. 1b). Particularly, among these compounds, HCJA121 and HCJA404 performed the best, promoting melanogenesis in a dose-dependent manner, with $\mathrm{EC}_{50}$ values of $4.68 \mu \mathrm{M}$ and $7.12 \mu \mathrm{M}$, respectively, which were considerably better than that of 8-MOP $\left(\mathrm{EC}_{50}=24.36 \mu \mathrm{M}\right)$. Therefore, HCJA121 and HCJA404 were deemed suitable for further bioassays, and $20 \mu \mathrm{M}$ was chosen as an effective treatment concentration. The effect of HCJA121 and HCJA404 on tyrosinase was measured by L-DOPA oxidation. Compared with treatment with DMSO alone (untreated condition), treatment with $20 \mu$ M HCJA121 and HCJA404 resulted in an increase in tyrosinase activity in the B16 cells (Fig. 1c), which suggested that HCJA121 and HCJA404 exhibit good potential for improving vitiligo symptoms.

\section{Effects of phenanthridine derivatives on melanogenic protein in B16 cells}

According to the regulatory networks underlying melanogenesis, upregulation of Wnt/ $\beta$-catenin signalling promotes the expression of a critical melanogenic protein, melanocyte-inducing transcription factor (MITF), which subsequently activates TYRP (tyrosinase-related protein) 1 and 2 and TYR (tyrosinase) genes and finally increases melanogenesis (Fig. 1d) (Liu et al., 2019; Manga, Sheyn, Yang, Sarangarajan \& Boissy, 2006). MITF controls the production of the pigment melanin, and TYRP1 and 2 are two transmembrane proteins. TYRP-1 has been reported to influence TYR activity via forming a complex with it and/or stabilizing it. TRP-2 functions as a dopachrome tautomerase downstream of TYR in the melanogenic pathway and regulates the quantity and quality of melanin produced during melanin biosynthesis. (Wang et al., 2017). As phenanthridine derivatives could significantly increase melanin synthesis, it is necessary to further explore whether and how phenanthridine derivatives affect the expression of MITF, TYRP 1 and 2, and TYR (tyrosinase). As shown in Fig. 1e, after $48 \mathrm{~h}$ of treatment with HCJA121 and HCJA404 $(20 \mu \mathrm{M})$, the MITF gene levels were significantly enhanced in the treatment groups. Simultaneously, the levels of the TYRP-1, TYRP-2, and TYR genes were significantly increased in the administration groups . Particularly, HCJA121 treatment resulted in the strongest activation of MITF, TYRP-1, and TYR, the levels of which were several-fold higher than those of the control. To further elucidate the effect of HCJA121 on the expression of melanogenic proteins (MITF TYRP-1, MITF TYRP-2, and MITF TYR) at the protein level, western blotting was performed using lysates of B16 murine melanoma cells that were treated with different doses of HCJA121. As presented in Fig. 1f, compared to the control treatment, HCJA121 treatment clearly increased the expression of MITF TRP-1 and TRP-2 by in a dose-dependent manner in the B16 cells. These results indicated that HCJA121 and HCJA404 could simultaneously upregulate all key melanogenic proteins, which suggested that these compounds may be upstream regulators of the MITF-TYRP/TYR melanogenic pathway.

\section{Correlation between melanogenesis and Wnt activation}

According to the results of the aforementioned bioassays, we concluded that phenanthridine derivatives could truly promote melanogenesis and significantly upregulate the expression of melanogenic proteins. Because these compounds have been demonstrated to be potent Wnt agonists and because the Wnt signalling pathway plays an important role in melanogenesis regulation, the correlation between melanogenesis and Wnt activation were further investigated. To investigate whether Wnt was upregulated while melanogenesis was promoted, the effect of HCJA121 and HCJA404 on endogenous Wnt target genes was initially examined in B16 cells. The results indicated that the compounds induced a dose-dependent increase in the expression of AXIN2 and DKK1 (Hoverter \& Waterman, 2008) in B16 cells and that there was a positive correlation between Wnt activation and melanogenesis promotion. In addition, since these compounds are Wnt ligand-dependent agonists, we hypothesized that they would be more efficient in B16 cells treated with Wnt3a-conditioned media than in B16 cells that were not been treated with Wnt3a-conditioned media. The 
data showed that HCJA121 and HCJA404 increased the levels of the melanogenesis gene to a lesser extent in the cells without Wnt3a-conditioned media than in those treated with Wnt3a-conditioned media (Fig. 2a), which suggested that these derivatives increase melanogenesis by activating Wnt.

To further confirm that phenanthridine derivatives could promote melanin synthesis through specific activation of Wnt, Xav939, a reported Wnt inhibitor ${ }^{30}$ (Huang et al., 2009), was added to drug-treated B16 cells. XAV939 treatment decreased the expression of melanogenesis markers (MITF , TYR , TRP1 , and TRP2 ) (Fig. 2b), antagonizing phenanthridine-derivative-mediated promotion of melanogenesis and tyrosinase activity (Fig. 2c). These preliminary results indicated that HCJA121 and HCJA404 truly promote melanogenesis by activating the Wnt signalling pathway.

\section{Anti-vitiligo mechanism and the target protein of phenanthridine derivatives}

To facilitate the identification of the cellular target (or targets) of HCJA121 and HCJA404, biotinylated derivatives were generated through label-free quantitative proteomic approaches. Axin appeared to act as a binding protein of HCJA121 and HCJA404, which was confirmed using a pull-down assay. Next, to confirm the anti-vitiligo target protein of the phenanthridine derivatives, we performed modelling studies for the complexes axin-HCJA121 and axin-HCJA404. A possible binding site for 1 and $\mathbf{2}$ on DAX, which has important implications on the function of axin, has been investigated using the AutoDock docking program (Morris et al., 2009) with the structure of DAX (PDB, 1WSP) (Schwarz-Romond et al., 2007) as the receptor molecule. Nine conformers of HCJA121 and 11 conformers of HCJA404 that were outputted from AutoDock onto DAX were analysed; six (HCJA121) and five (HCJA404) of the conformers were localized to the same site formed by the juxtaposition of the residues from two neighbouring protomers. Subsequently, the model with the lowest estimated free energy for binding (-9.35 kcal mol${ }^{-1}$ for HCJA121 and $-8.42 \mathrm{kcal} \mathrm{mol}^{-1}$ for HCJA404) was selected and modified slightly. The modelled structure of the complexes of axin with HCJA121 and HCJA404 revealed that LYS781 and LEU784 in the binding cavity could form contacts with HCJA121 and HCJA404 through electrostatic or hydrophobic interactions (Fig. 3a). To verify the importance of these residues in the binding of compounds, DAX mutants carrying K781A and L784A mutations were generated. As predicted by the model, in the two DAX mutants, HCJA121 and HCJA404 exhibited significantly reduced activation of not only Wnt target genes but also melanogenesis genes (Fig. 3b). Similar to melanogenesis, melanin synthesis was clearly decreased in drug-treated mutated cells (Fig. 3c). However, the effects of 8-MOP on melanogenesis genes and melanogenesis in mutated cells appeared to be unaffected, suggesting that compounds targeting the DAX domain of axin through binding with LYS781 and LEU784 potentiate the axin-LRP6 association to promote Wnt signalling transduction and eventually promote melanogenesis.

These data suggest that HCJA121 and HCJA404 could promote melanogenesis by activating the Wnt signalling pathway. We have previously suggested an autoinhibition hypothesis, according to which the binding of phenanthridine derivatives to the axin $\mathrm{C}$ terminus disrupts the interaction with the axin $\mathrm{N}$ terminus and thus relieves the autoinhibition of axin. Therefore, active axin binds LRP6 more easily and thus promotes LRP6 phosphorylation when the Wnt signalling pathway is activated ${ }^{24}$. Hence, we presumed that these anti-vitiligo phenanthridine derivatives could potentiate the axin-LRP6 association to influence melanin synthesis. To validate this hypothesis, we performed co-immunoprecipitation (co-IP) of axin and LRP6 from the lysates of HCJA121-treated B16 cells (using anti-axin1 and anti-LRP6 antibodies for immunoprecipitation); subsequently, the proteins were separated by western blotting. First, anti-axin1 was employed to capture the axin protein and axin-related complex. Next, LRP6 antibody was used on the protein mixtures captured by the axin1 antibody for capturing LRP6. The spot representing LRP6/anti-LRP6 from the HCJA121-treated group was considerably larger than that from the DMSO-treated group at the same loading quantity of the sample (Fig. 3d), which suggested that axin and LRP6 exhibited strong association in the phenanthridine-derivative-treated cells. The protein complex of axin and LRP6 in drug-treated cells appeared to be more common than it was in the control, which implies that phenanthridine derivatives could potentiate the axin-LRP6 association.

Pharmacodynamic evaluation of phenanthridine derivatives in c57bl/6 mice 
According to the aforementioned in vitro assays, HCJA121 and HCJA404 effectively stimulated melanin production through a new mode of action that involved targeting of the axin protein. Subsequently, in vivo pharmacodynamic assays were conducted to evaluate the therapeutic effects of HCJA121 and HCJA404 on vitiligo. We used a total of $108 \mathrm{C} 57 \mathrm{BL} / 6$ mice (half male and half female), weighing between 18 and $22 \mathrm{~g}$. We coated 99 mice with $5 \%$ HQ for 50 consecutive days to establish a mouse model of vitiligo. The mice were randomly divided into 8 groups: a negative control group, a positive control group (8-MOP), and six groups that were treated with low $(0.0425 \mathrm{mg} / \mathrm{kg})$, medium $(0.425 \mathrm{mg} / \mathrm{kg})$, or high $(4.25 \mathrm{mg} / \mathrm{kg})$ doses of the compounds. The remaining eight mice were not treated with $5 \% \mathrm{HQ}$, and they served as a blank control group. Phenanthridine was administered once daily for 30 days. During the modelling process, the skin of each group of mice was pale, and hair growth was slow. With prolonged modelling, white spots appeared, and the new hair turned white. As shown in Fig. 4a and Table S1, the skin and colour of the newly generated hair in the test area of the vitiligo mice exhibited varying degrees of restoration to black in the groups treated with 8-MOP and the phenanthridine derivatives. Particularly, the skin and hair of vitiligo mice recovered very well after the administration of HCJA121 and HCJA404, even at a low dose, indicating that the clinical symptoms of vitiligo mice were significantly improved, implying that 1 and 2 produce strong curative effects for vitiligo.

\section{Effects of phenanthridine derivatives on skin tissue of HQ-treated C57bl/6 mice}

After the pharmacodynamic evaluation, all experimental mice were euthanized, and the skin of the test area was removed, stained with haematoxylin eosin (HE), and observed under a microscope. The tyrosinase in the blood of drug-treated mice recovered remarkably and the MDA content decreased significantly in a dose-dependent manner (Fig 4b) compared to that in mice from the NC group. The results indicated that in comparison to the skin of the blank control, there were considerably fewer melanin granules and a thicker (10-20 layers of reticular structure) stratum corneum in the skin of the negative control. In contrast, skin samples from drug-treated groups displayed significantly different clinical features, with considerably more melanin granules and thinner (3-10 layers of reticular structure) stratum corneum (Fig. 4b). Similar to the aforementioned situation, the number of melanin granules in the skin of the compound-treated groups was significantly increased, even with a low treatment dose.

\section{Effects on melanin particle-containing hair follicles and epidermal cells in vitiligo mice}

Melanogenesis occurs in melanosomes, which are then transported to basal layer cells and hair follicles together with melanin. The amount of melanin in the normal skin is constant. Melanin is silver-compatible and argyrophilic. It can absorb ferrous ions, Lillie staining can show veins in a deep blue colour. Therefore, this staining method was selected to determine the number of epidermal cells containing melanin particles in each group under a light microscope. The average number of melanin-containing cells was calculated in every 100 basal cells and in every 50 hair follicles. The number of melanin-containing hair follicles in the back skin of the model group and the number of melanocytes in the basal layer were significantly decreased compared with that in the control group (Fig. 5a). The number of melanin-containing hair follicles in the skin and the number of basal cells were significantly increased (Fig. 5b), indicating that the compound induced a certain colour-reducing effect on the HQ-induced vitiligo mouse model, even at low doses.

\section{Toxicity text of phenanthridine derivatives in vivo}

To ensure host safety, ICR mice were treated once with HCJA121 and HCJA404 (intraperitoneally, at a dose of $2000 \mathrm{mg} / \mathrm{kg}$ ) and subsequently assessed for 14 days. None of the mice died in the phenanthridine-treated groups, and there was no change in body weight. Organs (liver, kidney, testis, and ovary) and blood samples collected at the end of the experiment were used for pathological and haematological examination. The results of the pathological analysis are shown in Fig. S2. No clear abnormalities were observed; however, there was slight vasodilatation and a low amount of lymphocyte distribution. The white cells and differential leukocyte parameters (Table S3) and erythrocytic parameters and platelet level parameters (Table S4) did not clearly change. It appears that these three compounds exhibit low acute toxicity; therefore, they can be considered safe according to this in vivo analysis. 


\section{Discussion}

Although activation of the Wnt/ $\beta$-Catenin signalling pathway has been linked to melanogenesis in melanocytes, no Wnt-based vitiligo treatment has been developed nor have relevant small molecule drugs been reported. In this study, we identified two phenanthridine-derived Wnt-specific agonists, HCJA121 and HCJA404, as potent anti-vitiligo agents. These compounds exhibited potent promotion of melanin synthesis and tyrosinase activity in B16 cells. Subsequent assays showed that melanogenic proteins, MITF, TYR, TRP1, and 2, were increased at both the mRNA and protein levels in the presence of HCJA121 and HCJA404, suggesting that these compounds may act as upstream regulators that modulate the MITF-TYRP/TYR melanogenic pathway by affecting Wnt. This speculation was then proven through co-administration of these compounds. In this study, the ability of the compounds to promote the expression of melanogenic proteins disappeared after the administration of XAV939, a classic Wnt/ $\beta$-Catenin inhibitor.

Analysis of the crystal structures of the axin protein and the structures of HCJA121 and HCJA404 revealed a potential ligand-binding pocket in DAX. Inside this pocket, there are two residues, LYS781 and LEU784, that could form contacts with HCJA121 and HCJA404 through electrostatic or hydrophobic interactions. The importance of LYS781 and LEU784 was further underscored by an in vitro reporter assay, which showed that the wild-type axin but not the mutant K781A or L784A could be induced by compounds to activate the Wnt target gene and, subsequently, the melanogenic genes. The mechanism can be concluded from the aforementioned results: HCJA121 and HCJA404 target axin via binding to LYS781 and LEU784 and subsequently potentiating the axin-LRP6 association to activate Wnt signalling transduction and eventually promote melanogenesis.

In vivo assays demonstrated that administration of the phenanthridine-derived compounds (even at a low dose) in HQ-treated C57BL/6 vitiligo mice could significantly restore skin lesions induced by HQ and improve vitiligo-related biochemistry indexes. The curative effects on vitiligo were better in the treatment group than in the control group. In addition, ICR mice were treated with HCJA121 and HCJA404 at a high dose for evaluating the safety of the compounds, and no abnormal change was observed in the body weight, organs, or blood samples of the mice.

In summary, this work demonstrates that phenanthridine compounds could potentiate the axin-LRP6 association to activate Wnt signalling transduction and eventually induce melanogenic protein expression by targeting the axin protein. These findings suggest a new therapeutic strategy for vitiligo. Importantly, HCJA121 and HCJA404 may represent potential candidates for vitiligo treatment.

\section{Acknowledgements}

We express great respect to all experimental animals that contributed to this research. This research was funded by the National Natural Science Foundation of China (81773610, 81973212); the Youth Innovation Promotion Association of CAS (2018429); the Central Asian Drug Discovery and Development Center of Chinese Academy of Sciences (CAM201801).

Open research (DATA AVAILABILITY STATEMENT)

The data that support the findings of this study are available from the corresponding author upon

reasonable request. Some data may not be made available because of privacy or ethical

restrictions.

Declaration of transparency and scientific rigour

This Declaration acknowledges that this paper adheres to the principles for transparent reporting and

scientific rigour of preclinical research as stated in the BJP guidelines for Design and Analysis, and

Animal Experimentation, and as recommended by funding agencies, publishers and other

organisations engaged with supporting research. 
Open research (DATA AVAILABILITY STATEMENT)

The data that support the findings of this study are available from the corresponding author upon reasonable request. Some data may not be made available because of privacy or ethical restrictions.

Declaration of transparency and scientific rigour

This Declaration acknowledges that this paper adheres to the principles for transparent reporting and scientific rigour of preclinical research as stated in the BJP guidelines for Design and Analysis, and Animal Experimentation, and as recommended by funding agencies, publishers and other organisations engaged with supporting research.

\section{References}

Anonymous (2015). Patient Perspectives: What is Vitiligo? Pediatr Dermatol 32: e328-329.

Boniface K, Seneschal J, Picardo M, \& Taieb A (2018). Vitiligo: Focus on Clinical Aspects, Immunopathogenesis, and Therapy. Clin Rev Allergy Immunol 54: 52-67

Boisseau-Garsaud AM, Garsaud P, Cales-Quist D, Helenon R, Queneherve C, \& Claire RC (2000). Epidemiology of vitiligo in the French West Indies (Isle of Martinique). Int J Dermatol 39: 18-20.

Curtis, M J, Alexander, S, Cirino, G, Docherty, J R, George, C H, Giembycz, M A, \& Ahluwalia, A. (2018). Experimental design and analysis and their reporting II: Updated and simplified guidance for authors and peer reviewers. British Journal of Pharmacology, 175: 987-993.

Cohen BE, Elbuluk N, Mu EW, \& Orlow SJ (2015). Alternative Systemic Treatments for Vitiligo: A Review. Am J Clin Dermatol 16: 463-474.

Clevers H, \& Nusse R (2012). Wnt/ $\beta$-catenin signaling and disease. Cell (Cambridge, MA, U S) 149: 1192-1205.

Chen D, Zhang H, Jing C, He X, Yang B, Cai J, et al . (2018). Efficient synthesis of new phenanthridine Wnt/ $\beta$-catenin signaling pathway agonists. Eur J Med Chem 157: 1491-1499.

Chen D-z, Yang B-j, He X-l, Fan S-r, Cai J-y, Jing C-x, et al . (2019). Design, synthesis and structureactivity relationship optimization of phenanthridine derivatives as new Wnt/ $\beta$-catenin signalling pathway agonists. Bioorg Chem 84: 285-294.

Chen D-Z, Jing C-X, Cai J-Y, Wu J-B, Wang S, Yin J-L, et al . (2016). Design, Synthesis, and Structural Optimization of Lycorine-Derived Phenanthridine Derivatives as Wnt/ $\beta$-Catenin Signaling Pathway Agonists. J Nat Prod 79: 180-188.

Ezzedine K, Eleftheriadou V, Whitton M, \& van GN (2015). Vitiligo. Lancet 386: 74-84.

Ezzedine K, Lim HW, Suzuki T, Katayama I, Hamzavi I, Lan CCE, et al . (2012). Revised classification/nomenclature of vitiligo and related issues: the Vitiligo Global Issues Consensus Conference. Pigment Cell Melanoma Res 25: E1-13.

Elbuluk N, \& Ezzedine K (2017). Quality of Life, Burden of Disease, Co-morbidities, and Systemic Effects in Vitiligo Patients. Dermatol Clin 35: 117-128.

Hayashi M, Jin Y, Ferrara TM, Spritz RA, Yorgov D, Santorico SA, et al . (2016). Autoimmune vitiligo is associated with gain-of-function by a transcriptional regulator that elevates expression of HLA-A*02:01 in vivo. Proc Natl Acad Sci U S A 113: 1357-1362.

Hoverter NP, \& Waterman ML (2008). A Wnt-fall for gene regulation: repression. Sci Signaling 1. 
Huang S-MA, Mishina YM, Liu S, Cheung A, Stegmeier F, Michaud GA, et al . (2009). Tankyrase inhibition stabilizes axin and antagonizes Wnt signalling. Nature (London, U K) 461: 614-620.

Lopes C, Trevisani VFM, Trevisani VFM, Trevisani VFM, Melnik T, \& Melnik T (2016). Efficacy and Safety of 308-nm Monochromatic Excimer Lamp Versus Other Phototherapy Devices for Vitiligo: A Systematic Review with Meta-Analysis. Am J Clin Dermatol 17: 23-32.

Li L, Liang Y, Zhang D, Wang C, Pan N, Hong J, et al . (2019). The 308-nm excimer laser stimulates melanogenesis via the wnt/ $\beta$-Catenin signaling pathway in B16 cells. J Dermatol Treat 30: 826-830.

Lerner AB, Denton CR, \& Fitzpatrick TB (1953). Clinical and experimental studies with 8-methoxypsoralen in vitiligo. J Invest Dermatol 20: 299-314.

Liu L-P, Li Y-M, Guo N-N, Li S, Ma X, Zhang Y-X, et al . (2019). Therapeutic Potential of Patient iPSC-Derived iMelanocytes in Autologous Transplantation. Cell Rep 27: 455-466.e455.

Manga P, Sheyn D, Yang F, Sarangarajan R, \& Boissy RE (2006). A role of tyrosinase-related protein 1 in 4-tert-butylphenol-induced toxicity in melanocytes: implication for vitiligo. Am J Pathol 169: 1652-1662.

Morris GM, Huey R, Lindstrom W, Sanner MF, Belew RK, Goodsell DS, et al . (2009). AutoDock4 and AutoDockTools4: Automated docking with selective receptor flexibility. J Comput Chem 30: 2785-2791.

Niezgoda A, Nowowiejska L, Bialecka A, Mecinska-Jundzill K, Adamska U, Czajkowski R, et al . (2017). Properties of skin stem cells and their potential clinical applications in modern dermatology. Eur J Dermatol 27: 227-236.

Olsen JR, Gallacher J, Finlay AY, Piguet V, \& Francis NA (2016). Quality of life impact of childhood skin conditions measured using the Children's Dermatology Life Quality Index (CDLQI): a meta-analysis. Br J Dermatol 174: 853-861.

Picardo M, Dell'Anna ML, Ezzedine K, Taieb A, Hamzavi I, Harris JE, et al . (2015). Vitiligo. Nat Rev Dis Primers 1: 15011.

Rashighi M, \& Harris JE (2017). Vitiligo Pathogenesis and Emerging Treatments. Dermatol Clin 35: 257-265.

Rodrigues M, Ezzedine K, Hamzavi I, Pandya AG, \& Harris JE (2017). Current and emerging treatments for vitiligo. J Am Acad Dermatol 77: 17-29.

Sehgal VN, \& Srivastava G (2007). Vitiligo: compendium of clinico-epidemiological features. Indian J Dermatol Venereol Leprol 73: 149-156.

Schwarz-Romond T, Fiedler M, Shibata N, Butler PJG, Kikuchi A, Higuchi Y, et al . (2007). The DIX domain of Dishevelled confers Wnt signaling by dynamic polymerization. Nat Struct Mol Biol 14: 484-492.

Wang S, Yin J, Chen D, Nie F, Song X, Fei C, et al . (2013). Small-molecule modulation of Wnt signaling via modulating the Axin-LRP5/6 interaction. Nat Chem Biol 9: 579-585.

Wang JY, Chen H, Wang YY, Wang XQ, Chen HY, Zhang M, et al . (2017). Network pharmacological mechanisms of Vernonia anthelmintica (L.) in the treatment of vitiligo: isorhamnetin induction of melanogenesis via up-regulation of melanin-biosynthetic genes. BMC Syst Biol 11: 103/101-103/112.

Yamada T, Hasegawa S, Inoue Y, Date Y, Yamamoto N, Mizutani H, et al . (2013). Wnt/B-Catenin and Kit Signaling Sequentially Regulate Melanocyte Stem Cell Differentiation in UVB-Induced Epidermal Pigmentation. J Invest Dermatol 133: 2753-2762.

Zeng X, Tamai K, Doble B, Li S, Huang H, Habas R, et al . (2005). A dual-kinase mechanism for Wnt co-receptor phosphorylation and activation. Nature (London, U K) 438: 873-877. 
Zimmerman ZF, Moon RT, \& Chien AJ (2012). Targeting Wnt pathways in disease. Cold Spring Harbor Perspect Biol 4: a008086/008081-a008086/008024.

\section{Author Contributions}

+ Duo-zhi Chen, Shirui Fan, and Xueyin Lu contributed equally to this work. D. C., C. J., and B. Y. designed and performed the chemical synthesis; J. C. and Y. W. purified all the compounds; S. F., X. L., D. Z., and Y. Y. evaluated the antiviral activities and other bioactivities of all the compounds under the guidance of A. H. and L. L.; D. C. and S. F. wrote the manuscript with advice from all of the authors; and X. H. and D. C. guided all aspects of this work.

\section{Competing Interests' Statement}

The authors declare no competing interests.

a.

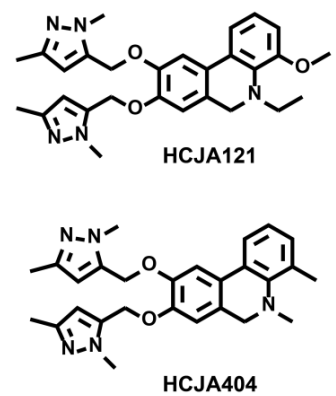

d.

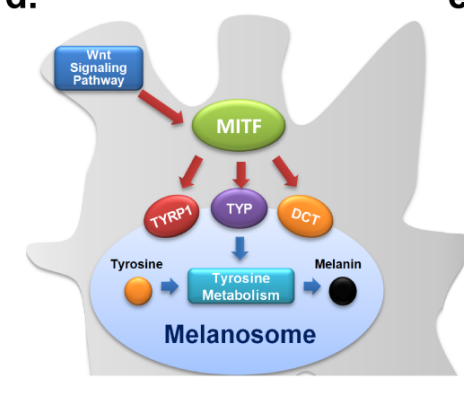

f.

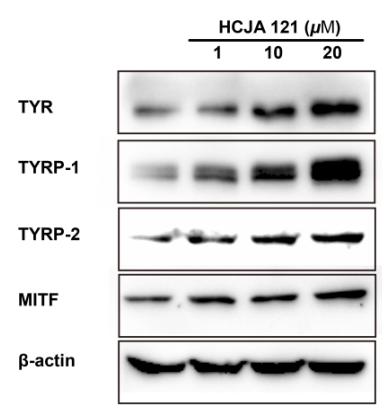

. b.

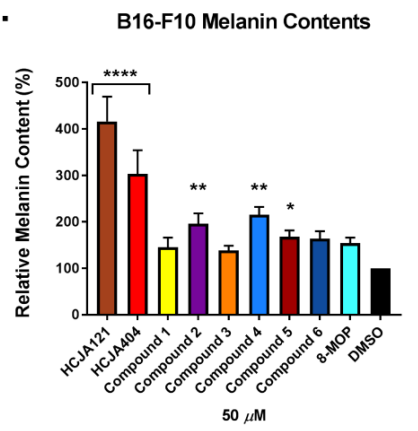

e.

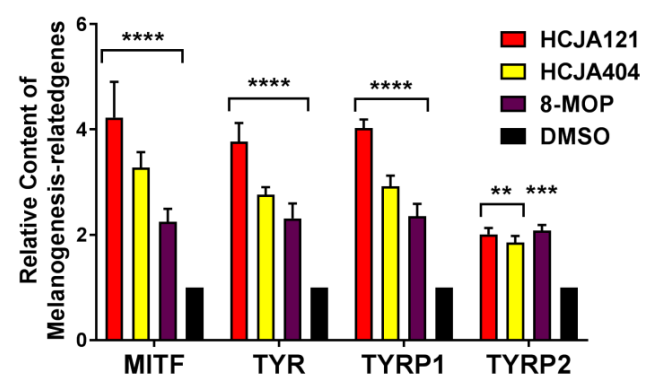

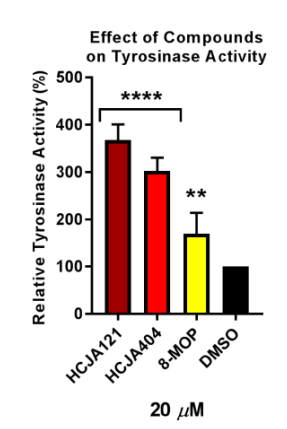

$20 \mu \mathbf{M}$ 
nm. The melanin content was calculated relative to that in the control group and was corrected on the basis of the protein concentrations, considering the control group as representing $100 \%$ melanin content. (c) HCJA121 and HCJA404 $(20 \mu \mathrm{M})$ significantly increased tyrosinase activity in B16-F10 cells. The cellular tyrosinase activity was estimated by measuring the rate of L-3,4-dihydroxyphenylalanine (L-DOPA) oxidase activity. The tyrosinase activity of each sample was calculated and corrected for the concentrations of proteins, considering the control group as representing $100 \%$ activity. (d) The MITF regulatory network underlying melanogenesis. (e) HCJA121 and HCJA404 increased the expression of the MITF, TYR, and TYRP 1 and2 genes. B16 cells pre-treated with HCJA121, HCJA404, or 8-MOP for $2 \mathrm{~h}$ were incubated with Wnt3a-conditioned medium containing the same dose of compounds for an additional $48 \mathrm{~h}$. MITF , $T Y R$, and TYRP 1 and 2 were analysed by $\mathrm{qPCR}$. The results were normalized to $\beta$-actin expression. (f) Compound HCJA121 increases the levels of the MITF, TYR TYRP 1, and TYR TYRP 2 proteins. B16 cells treated with HCJA121 for $48 \mathrm{~h}$ were fractionated as described in the Methods section and subjected to western blot analysis. $\beta$-actin served as the loading control. The immunoblots were quantified by densitometry, and the intensity values were normalized to $\beta$-actin. The values are indicated beneath each band. The data shown represent means \pm standard deviations (S.E.M) from five independent experiments $(\mathrm{n}=5)$. Significantly different as indicated $\left({ }^{*} P<0.05,{ }^{* *} P<0.01,{ }^{* * *} P<0.001\right.$, and $\left.{ }^{* * * *} P<0.0001\right)$. 
a.

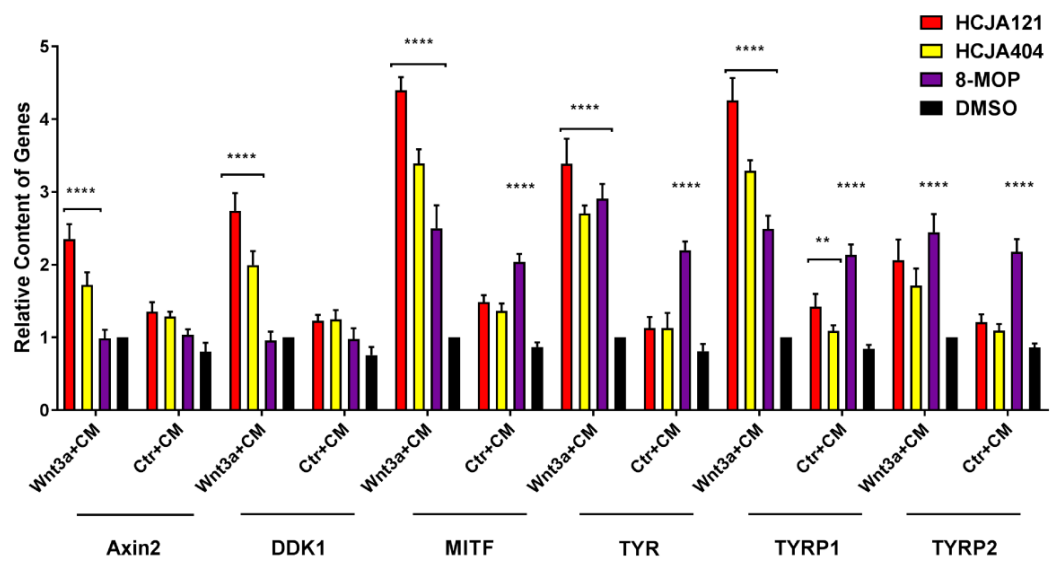

b.

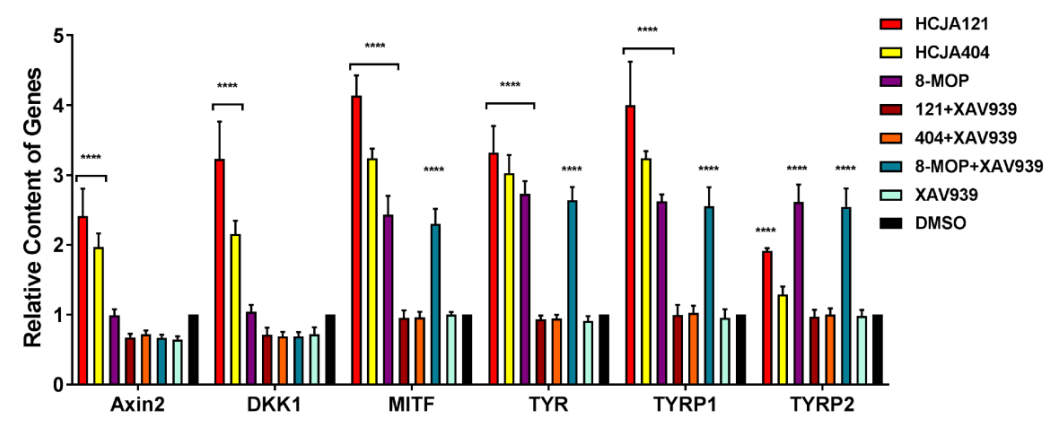

c.
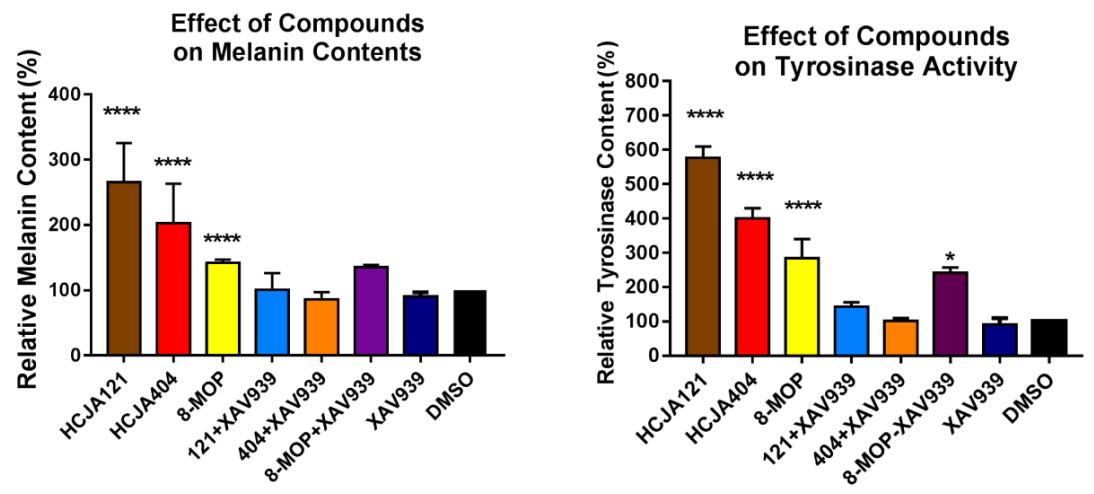

Figure 2. Wnt/ $\beta$-catenin signalling mediates melanogenesis, according to a correlation analysis performed in B16 cells. (a) Comparison of the effects of HCJA121 and HCJA404 on increasing the expression of the Axin2 , DKK1 , MITF ,TYR , and TYRP 1 and 2 genes in cells treated with Wnt3a-conditioned media and those that were not treated with Wnt3a-conditioned media. (b) Treatment with $20 \mu$ M HCJA121 and HCJA404 increased the expression of the Axin2 , DKK1 ,MITF , TYR, and TYRP 1 and 2 genes with 8-MOP as the positive control, which was abolished by complementation with $5 \mu$ M XAV939, a Wnt inhibitor. B16 cells were treated with the compounds or compounds in combination with XAV939 for $48 \mathrm{~h}$, and melanogenesis was determined by qPCR. (c) The induction of melanin content and tyrosinase activity by HCJA121 and HCJA404 $(20 \mu \mathrm{M})$ was blocked by $5 \mu \mathrm{M}$ Wnt inhibitor XAV939. The results represent the means \pm S.E.M from five independent experiments $(n=5)$. Data were analysed by ANOVA, significantly 
different as indicated $\left({ }^{*} P<0.05,{ }^{* *} P<0.01,{ }^{* * *} P<0.001\right.$, and $\left.{ }^{* * * *} P<0.0001\right)$.
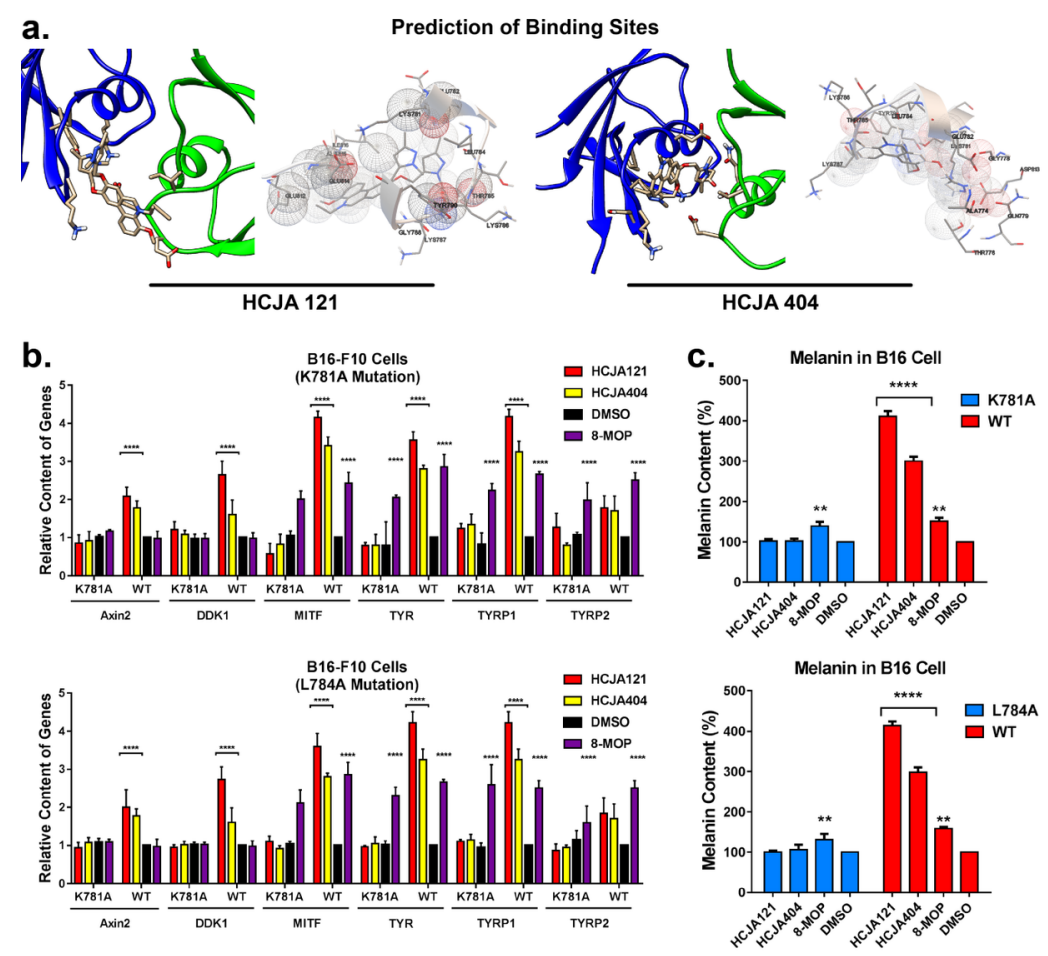

d.
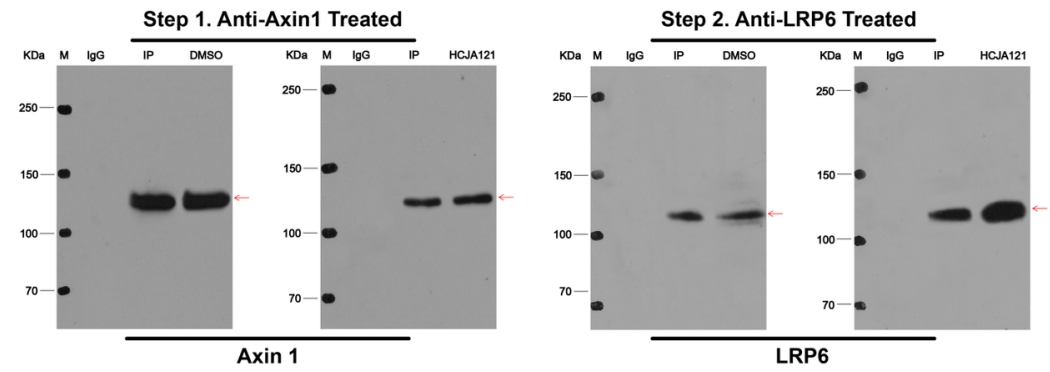

Figure 3. Axin is the target protein of HCJA121 and HCJA404. (a) A cartoon representation of HCJA121 (sapphire sticks) and HCJA404 (purple sticks) docked onto the crystal structure of the DAX domain of axin (PDB 1WSP). The related two protomers of DAX are in blue and green, respectively. b) K781A or L784A mutations within the DAX domain showed decreased acceleration of melanogenesis following treatment with compounds HCJA121 and HCJA404 compared to their effects in wild-type (WT) B16 cells. (c) Stimulation of melanin content and tyrosinase activity by HCJA121 and HCJA404 (20 $\mu$ M) in K781A or L784A mutated B16 cells was significantly decreased relative to that in the control cells. The results represent the means \pm S.E.M from five independent experiments $(\mathrm{N}=5)$. Data were analysed by ANOVA, significantly different as indicated. (d) Co-IP of axin and LRP6 from B16 cells implies that the compounds could enhance the interaction between axin and LRP6. The protein was first precipitated using an axin antibody and subsequently with an LRP6 antibody. The amount of each sample assessed by WB was $50 \mu$ g. Data shown are means \pm S.E.M; $\mathrm{n}=5$ per group. significantly different as indicated $\left({ }^{*} P<0.05,{ }^{* *} P<\right.$ $0.01,{ }^{* * *} P<0.001$, and $\left.{ }^{* * * *} P<0.0001\right)$. 
a.
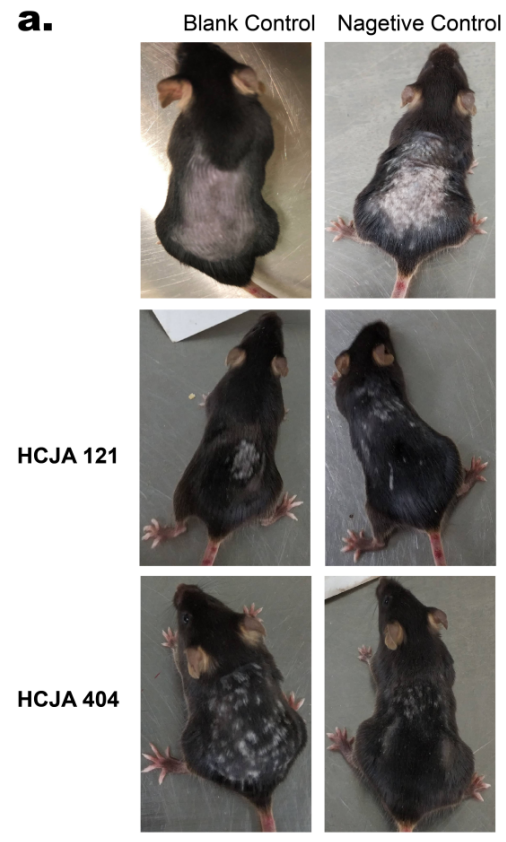

$0.0425 \mathrm{mg} / \mathrm{kg}$
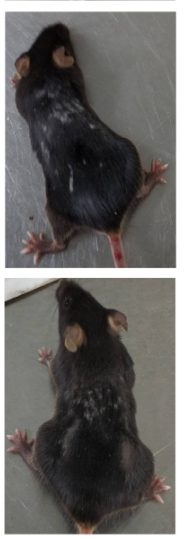

$0.425 \mathrm{mg} / \mathrm{kg}$
8-MOP
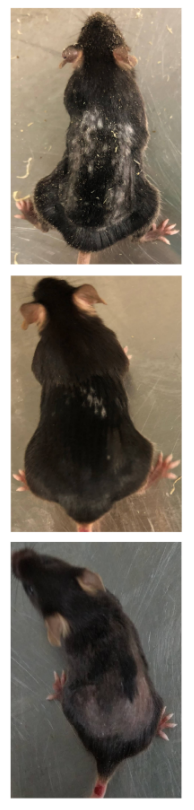

$4.25 \mathrm{mg} / \mathrm{kg}$ b.
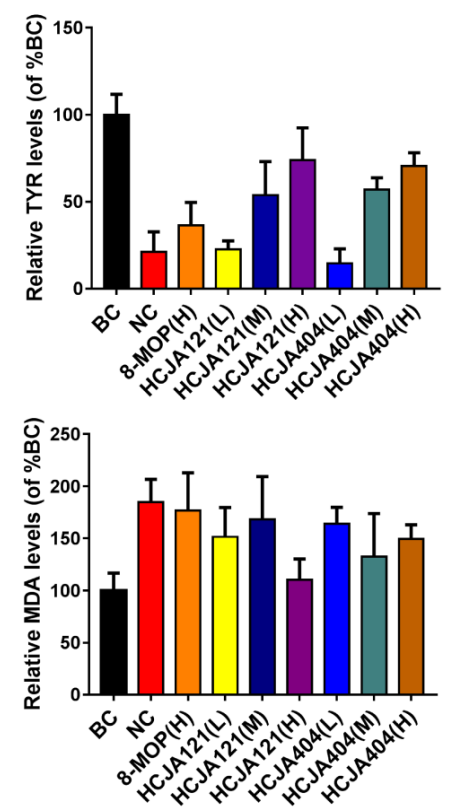

c.

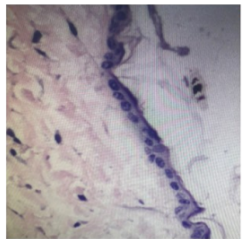

Blank Control
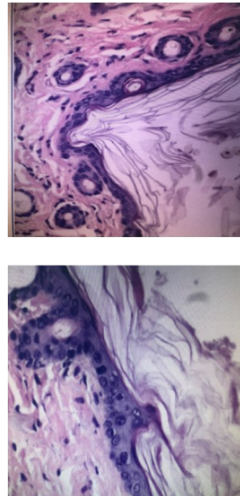

$0.0425 \mathrm{mg} / \mathrm{kg}$

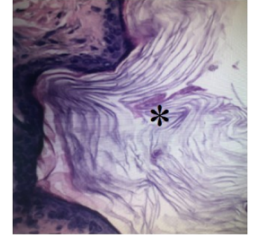

Nagetive Control
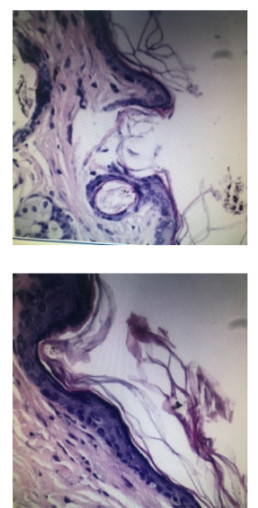

$0.425 \mathrm{mg} / \mathrm{kg}$

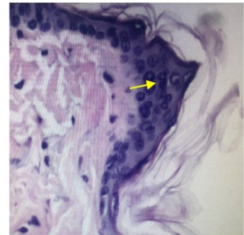

8-MOP
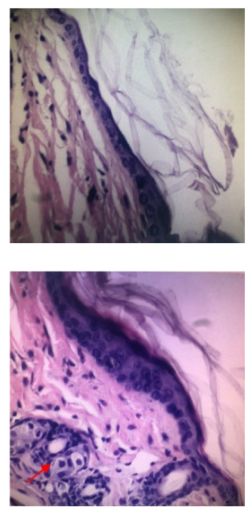

$4.25 \mathrm{mg} / \mathrm{kg}$

Figure 4 . Effects of phenanthridine derivatives on hydroquinone-treated C57BL/6 mice. As described in the Methods, the C57BL/6 mice were coated with $5 \%$ hydroquinone for 50 consecutive days to establish a mouse model of vitiligo. Subsequently, the animals were acclimatized for 1 week and randomly divided into eight groups, including one negative control group, one 8-MOP group, three HCJA121-treated groups, and three HCJA404-treated groups, each group has 12 mice $(\mathrm{n}=12)$. The 8-MOP group was administered 8-MOP $(4.25 \mathrm{mg} / \mathrm{kg})$, and the drug-treated groups were administered HCJA121 or HCJA404 at one of three doses (L: $0.0425 \mathrm{mg} / \mathrm{kg}, \mathrm{M}: 0.425 \mathrm{mg} / \mathrm{kg}$ and H: $4.25 \mathrm{mg} / \mathrm{kg}$ ). In addition, 12 normal mice were established as the 
blank control. At the end of the 30-day treatment, the blood was collected retro-orbitally from the mice, and dorsal skin samples were isolated from the animals and stained with HE. (a). Recovery effects of phenanthridine derivatives on skin lesions in hydroquinone-treated C57BL/6 mice; (b). Compared to mice from the $\mathrm{NC}$ group, the tyrosinase in the blood of the drug-treated mice recovered remarkably, and the MDA content decreased significantly. (c) Skin histomorphology changes in the treated mice are presented. Photomicrography indicated that HCJA121 and HCJA404 are excellent for promoting the repair of hydroquinone-damaged melanin in epidermal basal layer cells. The asterisk indicates the thickened stratum corneum, the black arrow indicates a layer of darker-purple granules, the yellow arrow indicates the increased spinal cell layer in the epidermal layer, and the red arrows indicate the hair follicle sebaceous glands; magnification, $200 \times$. Data shown are means \pm S.E.M; $\mathrm{n}=12$ mice per group. Significantly different as indicated $\left({ }^{*} P<0.05,{ }^{* *} P\right.$ $<0.01,{ }^{* * *} P<0.001$, and $\left.* * * * P<0.0001\right)$. 
a.

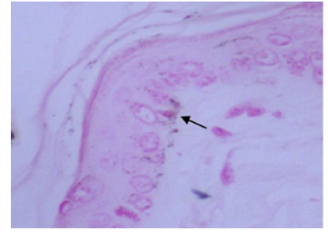

Blank Control

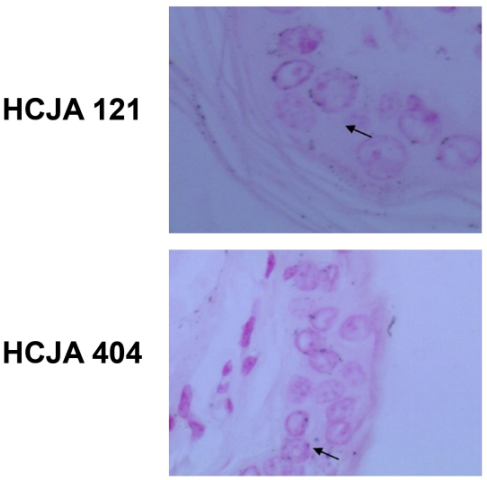

$0.0425 \mathrm{mg} / \mathrm{kg}$

b.

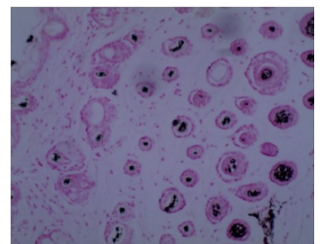

Blank Control
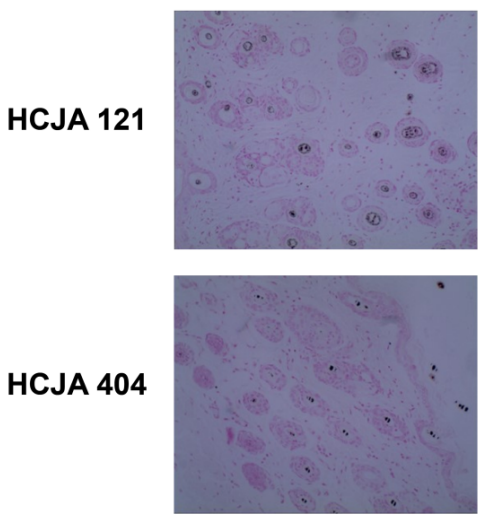

$0.0425 \mathrm{mg} / \mathrm{kg}$

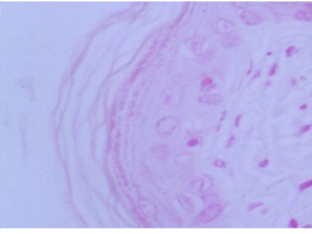

Nagetive Control
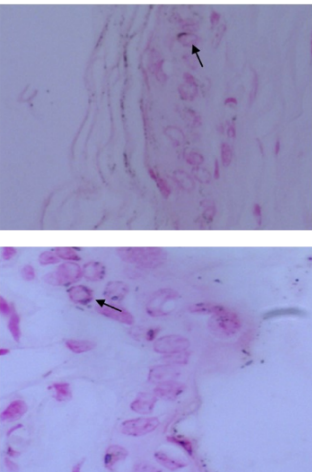

$0.425 \mathrm{mg} / \mathrm{kg}$

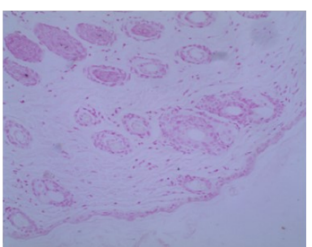

Nagetive Control
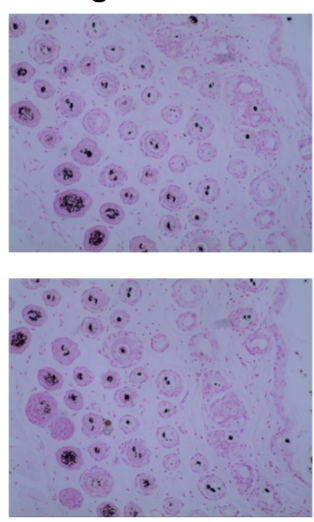

$0.425 \mathrm{mg} / \mathrm{kg}$

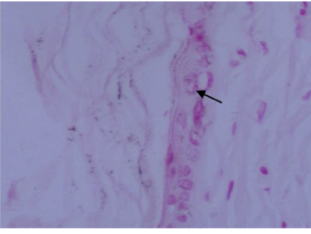

8-MOP
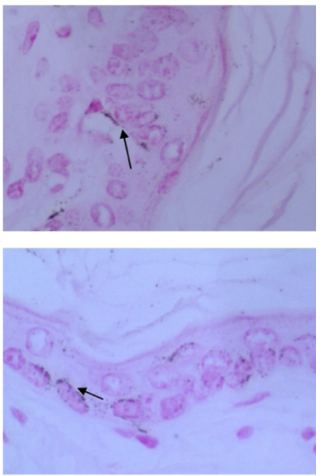

$4.25 \mathrm{mg} / \mathrm{kg}$

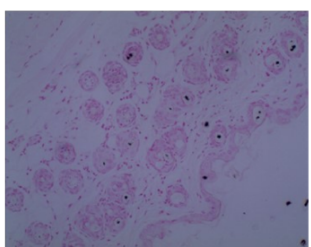

8-MOP
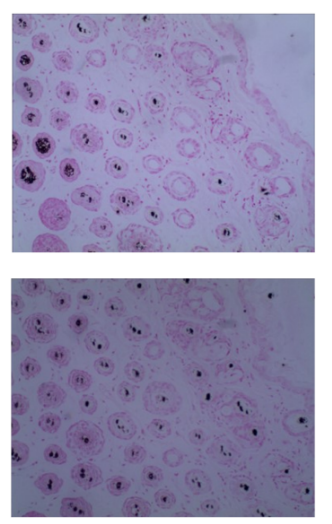

$4.25 \mathrm{mg} / \mathrm{kg}$

Figure 5. Melanin-particle-containing hair follicles and epidermal cells in Vitiligo mice. a). HCJA121 and HCJA404 significantly increased the number of melanin-containing epidermal cells in the skin of vitiligo mice. The black arrows indicate epidermal cells that contain melanin particles; magnification, $200 \times$; b). The compounds could clearly increase the melanin-particle-containing hair follicles in the skin of vitiligo mice; magnification, $200 \times . \mathrm{n}=12$ mice per group. 